\title{
Matching games with partial information
}

\author{
Paolo Laureti ${ }^{1}$, Yi-Cheng Zhang \\ Institut de Physique Théorique, Université de Fribourg, Pérolles, CH-1700 \\ Fribourg, Switzerland
}

\begin{abstract}
We analyze different ways of pairing agents in a bipartite matching problem, with regard to its scaling properties and to the distribution of individual "satisfactions". Then we explore the role of partial information and bounded rationality in a generalized Marriage Problem, comparing the benefits obtained by self-searching and by a matchmaker. Finally we propose a modified matching game intended to mimic the way consumers' information makes firms to enhance the quality of their products in a competitive market.
\end{abstract}

Key words: Matching Problems; Game theory; Bounded rationality. PACS: 05.20.-y; 01.75.+m; 02.50.Le;

\section{Marriage model as a market metaphor}

The Marriage Problem [1] describes a system where two classes of $N$ players, that we shall often call men and women, can be matched pairwise to their mutual benefit. In different contexts players could be producers and consumers, employers and job seekers or resources and activities in general. In fact, while the metaphorical model of marriages between men and women is suggestive, the importance of studying matching models lies, for us, in their broad implications in economic and social contexts. The fundamental premise is that there are many mutually beneficial relations out there to be found, like the one linking consumers with specific wishes to firms with suitable products. Mutually beneficial does not imply equally beneficial: previous work shows that, whoever processes more information, can in general reap more benefits $[2]$.

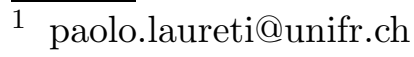

Preprint submitted to Elsevier Science 31 October 2018 
In this work we study further the matching model in some detail. In particular, we want to compare the matchmaker mediated matches (global optimum) with those obtained through the self-searching mode. The latter can be Nash equilibria [3] or satisficing matches, according to the definition of H. Simon [4]. He claims that people face uncertainty about the future and costs in acquiring information in the present. Sometimes information is simply unavailable. The best agents can do, with partial information and limited research capability, is setting an aspiration level which, if achieved, they will be happy enough with.

Even if agents were "maximizers" of some utility function, partial information would still prevent potential optimal partners from finding each other. Our analysis allows us to determine how good are the approximations agents must be content with, in the absence of complete information. This is particularly relevant in economic relations, notably in the law of Supply and Demand. Neoclassical economics generally assumes perfect information is available, leading to optimal solutions. More recent researches, like the one conducted by Akerlof [5], suggest that in extreme cases of asymmetric information, potentially beneficial transactions may fail to materialize.

However, when a transaction is still realized, even with limited information, standard economics literature does not address the problem of determining how far from the optimum it falls. We try here, as it has previously been done in a different context [6], to quantify the dependence of this distance from the amount of available information. Our approach offers an open-ended research agenda, aiming to give a micro detailed description of macroscopic matchings $[7]$.

\section{Average satisfaction in matching games}

Let us now formalize the model. We start with $2 N$ players, $N$ men and $N$ women, who are to be matched. Each player is been assigned his/her list of preferred partners. The lists are drawn at random and are independent from one another. If man $m$ marries woman $w$ we attribute him an energy equal to the ranking of $w$ in $m$ 's list. Let us define the matrices $f$ (for women) and $h$ (for men), such that the element $f(w, m)$ denotes the rank of man $m$ in $w$ 's list and $h(m, w)$ the rank of woman $w$ in $m$ 's list. The average energy per person in a given matching is defined by

$$
\epsilon=\frac{1}{N} \sum_{w=1}^{N} f\left(w, m_{\mathrm{w}}\right)+\frac{1}{N} \sum_{m=1}^{N} h\left(m, w_{\mathrm{m}}\right),
$$




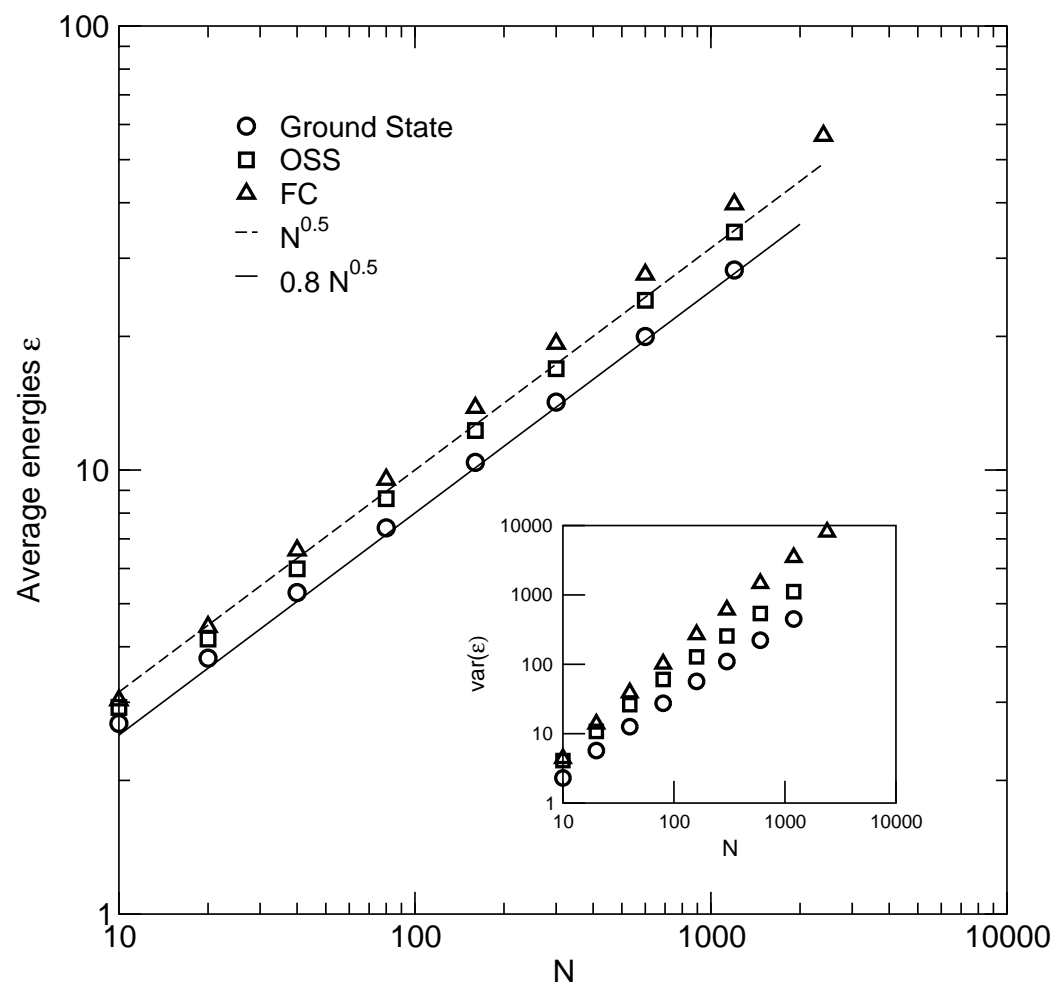

Fig. 1. Average energy of agents $\epsilon$ as a function of $N$ in $\log$-log scale. The dashed line is obtained from eq. (3) and the solid line from eq. (2). Symbols correspond to numerical calculations of the Ground State (circles), the OSS (squares) and the FC State (triangles), averaged over 1000 repetitions. In the inset graph we plotted their respective variances.

where $m_{\mathrm{w}}$ is the husband of woman $w$ and $w_{\mathrm{m}}$ the wife of man $m$. Low energy corresponds to high satisfaction and vice-versa.

There are many ways to match pairwise the $2 N$ players for a given instance of the preference lists, whether or not the notion of stability is taken into account [8]. If we treat the Marriage Problem as an optimization problem, then we can define the following relevant states:

- Ground State (state with the minimal total energy).

- Optimal Stable State (stable state with the minimal total energy).

The first one is globally optimal, the second is a constrained optimal state. Both of them are rather unlikely to be found in real life, because of incomplete information and limited searching power [9]. In particular men may not know all the women and vice-versa, or they may not dare to divorce once they found an acceptable partner. Following the lines traced in ref. [2] we propose a new procedure, the First Choice, that allows a selfish agent to find a suitable partner in a limited time. Here we shall mainly consider its symmetrical version, i.e. we make no difference between the strategies of men and women. 


\subsection{Ground State}

Often referred to as Assignment Problem [17], the problem of finding the matching of minimal energy in a bipartite graph has a long history in Operations Research. It has been shown that, in an instance of size $N$ of the marriage problem, the Hungarian algorithm [18] finds the Ground State in $O\left(N^{3}\right)$ steps. The average energy of the corresponding matching has been calculated analytically in reference [16]:

$$
\epsilon^{G S}=0.81 \sqrt{N}
$$

The above esteem (solid line) is compared with simulation data (circles) in figure 1, with good agreement.

\subsection{Optimal Stable State}

The Stable Marriage Problem [10,11] describes a complex system where individuals attempt to optimize their own satisfaction, subject to mutually conflicting constraints. A stable matching is defined by the property that there are no two couples $(m, w)$ and $\left(m^{\prime}, w^{\prime}\right)$, such that $h\left(m, w^{\prime}\right)<h(m, w)$ and $f\left(w, m^{\prime}\right)<f(w, m)$. The algorithm of Gale and Shapley [1] yields the stable matching with minimal average men's energy (male optimal). Starting from the male optimal solution, all other stable matchings can be obtained by properly performing cyclic exchange processes called rotations. If one wanted to find the stable matching that minimizes the total energy $\epsilon$, one could thus enumerate all stable matchings and compare their values. Unfortunately it has been shown that the maximum and the average number of stable matchings, for an instance of size $N$ of the preference lists, grow, respectively, as $e^{N}$ [12] and as $N \log (N)[11,13]$. In order to find a faster algorithm reference [14] suggests to construct a weighted directed graph of all rotations. The authors show that the maximum-weight closed subset of the rotation poset gives the OSS. The algorithm they propose scales as $N^{4}$, i.e. the running time of the Ford-Fulkerson algorithm [15] to find a minimum cut in the graph.

Since in stable matchings the average male and female energies are found to obey the relation $\epsilon_{\mathrm{m}} \epsilon_{f}=N[16]$, in a sex-fair matching, such as the OSS, the average energy per person must be

$$
\epsilon^{O S S}=\sqrt{N}
$$

In figure 1 the above equation, plotted as a dashed line, is shown to fit well 
the simulation data we obtained for the OSS (squares) using the algorithm described in [14].

\subsection{First Choice}

In the First Choice model (FC), at time step $n$ every player proposes to the $n^{\text {th }}$ of his/her preference list. Man $m$, for instance, will propose to woman $w$, such that $h(m, w)=n$. If $w$ is still unmarried, $f(w, m) \leq n$ and $w$ receives no better proposal at time step $n$, then she retains $m$ 's one and they get married. Marriages cannot be broken: married couples sit aside till the end of the game. If there are conflicting proposals the couples with the lowest energies are married first. When all the couples meeting this criteria at time step $n$ got married, bachelors propose the $(n+1)^{t h}$ of their preference lists, and so forth. The game proceeds till everybody is married, reaching what we shall call FC state. Since energy corresponds to number of proposals, the FC state can be found in less than quadratic time.

The results of our extensive simulations, reported in fig. 1, show the gap between the three different ways of modeling agents' behavior. The Ground State corresponds to the state of maximum global satisfaction, but there is no reason why selfish players should be able to find it by themselves, and not move from it once reached. Only an external institution, a matchmaker, could help people coordinate in such a profitable way. On the other hand, the OSS would not change, once reached by rational players. Since there is no procedure to reach it, though, the same rational players are much more likely to get trapped in another stable state. The OSS is, then, the best matching perfect agents could possibly obtain by self-searching. Numerical simulations of the FC state, reported as triangles up in figure 1, show that the FC matching gives average energies slightly above those of the OSS, and the same holds for their respective variances (inset graph). FC agents retain the best possibility they encounter at a given time and do not need to process all the virtually infinite information available. The same way consumers sometimes keep buying a specific, satisficing, product, instead of spending their time and money trying out all the other ones.

\section{Individual energy distribution with complete information}

We turn now our attention to single individuals, by tracing the individual energy distribution for the three matching methods. That of the OSS can be estimated following the same reasoning as in reference [13], where the authors define the ensemble of all stable matchings. In our case we just have to add 
the symmetry constraint $\epsilon_{f}=\epsilon_{m}$. Using continuous variables and rescaling the rankings such that $x_{\mathrm{m}} \simeq h\left(m, w_{\mathrm{m}}\right) / N, X=\sum_{i=1}^{N} x_{i}$ and $y_{\mathrm{w}} \simeq f\left(w, m_{\mathrm{w}}\right) / N$, $Y=\sum_{j=1}^{N} y_{j}$, the probability of finding a symmetrical stable state reads:

$$
\begin{aligned}
P & =\int_{0}^{1} d^{N} x \int_{0}^{1} d^{N} y \prod_{i \neq j}\left(1-x_{i} y_{j}\right) \delta\left(\sum_{i} x_{i}-\sum_{j} y_{j}\right) \\
& \simeq \int_{0}^{1} d^{N} x e^{-X^{2}} \rho_{N}^{2}(X)
\end{aligned}
$$

where [13]:

$$
\rho_{N}(X)=\int_{0}^{1} d^{N} x \delta\left(X-\sum_{i=1}^{N} x_{i}\right) \simeq \frac{X^{N-1}}{\Gamma(N)}\left(1-e^{-N / X}\right)^{N} .
$$

The single agent energy distribution can then be calculated by taking the average of $\delta\left(x-x_{N}\right)$ in the above ensemble, which reads:

$$
\begin{aligned}
<\delta\left(x-x_{N}\right)> & =\frac{1}{P} \int_{0}^{N} e^{-X^{2}} \rho_{N-1}(X-x) \rho_{N}(X) \\
& \simeq(N-1) \frac{(\sqrt{N}-x)^{N-2}}{N^{\frac{N-1}{2}}} \rightarrow \sqrt{N} e^{-x \sqrt{N}},
\end{aligned}
$$

for $N \gg x$. In fact the integral in (5) can be solved with the saddle point method around $X=\sqrt{N}$, where the factor $\left(1-e^{-N / X}\right)^{N}$ of equation (4) is almost equal 1. Hence the distribution of the average player's energy $\epsilon_{\mathrm{i}}=N x$ equals

$$
p\left(\epsilon_{\mathrm{i}}\right)=\frac{1}{\sqrt{N}} e^{-\frac{\epsilon_{\mathrm{i}}}{\sqrt{N}}} .
$$

The above becomes a simple exponential, if we rescale the $\epsilon_{\mathrm{i}}$ variable as follows:

$$
\tilde{\epsilon}_{\mathrm{i}}=\frac{\epsilon_{\mathrm{i}}}{\sqrt{N}}
$$

Equation (7), plotted as a solid line, agrees with the numerical simulations of the OSS reported in fig. 2.b, while it lies below the fat tail of the individual energy distribution of the FC State (fig. 2.a). Such a discrepancy is due to the fact that FC marriages are unbreakable, so the unlucky players married as last end up with energies comparable to that of a random choice $(N / 2)$. In real 


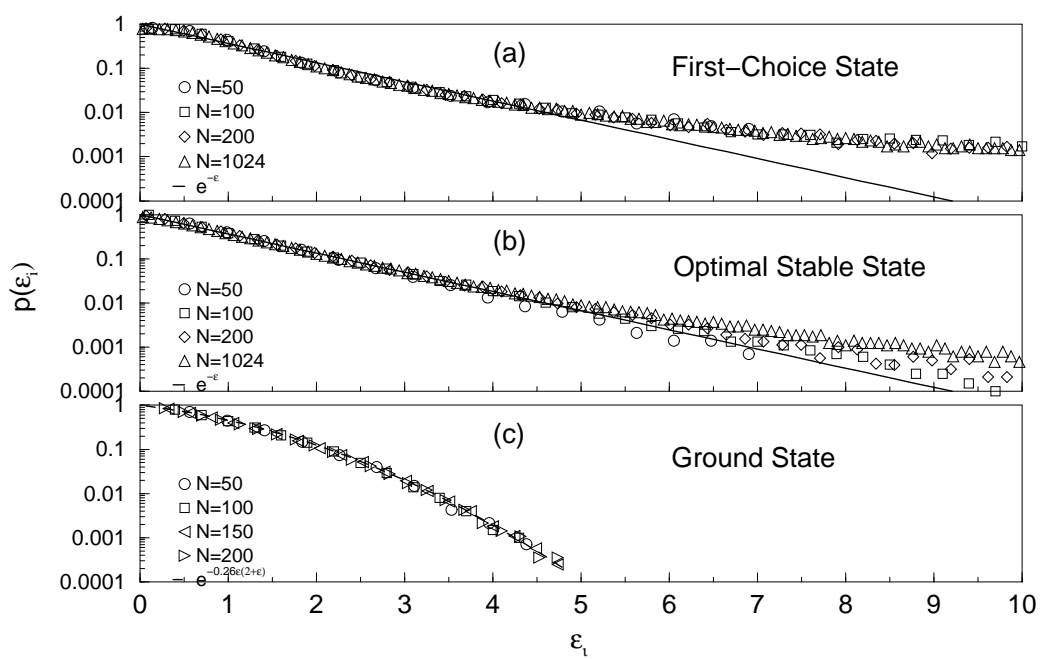

Fig. 2. Probability distribution of average individual energies $\epsilon_{\mathrm{i}}$, rescaled according to equation eq. (8), in the First Choice (a), Optimal Stable (b) and Ground State (c), plotted in linear-log scale. Symbols correspond to simulations obtained for different values of $N$, as reported in the legends. The solid lines represent the analytical solution for the OSS (equation (7)), the dashed line fits the data for the Ground State.

life social relations are not static, but local rearrangements, such as divorces or lay-offs, take place on a much longer time scale than that needed to find a partner.

The distribution of Ground State energies obeys perfectly the scaling of equation (8) and is well fitted by the Gaussian-like distribution $e^{-0.26 \epsilon_{\mathrm{i}}\left(2+\epsilon_{\mathrm{i}}\right)}$. This suggests that the globally optimal solution tends to assign similar satisfactions to every agent, balancing the loss of those who happen to be more "beautiful" [19] in a given instance of the preference lists with the gain of the least attractive ones. Such a fair and profitable matching, though, is prevented by the individual selfishness of agents endowed with perfect information. In the following sections we will show how things may change when individual information is incomplete.

\subsection{Evolutionary stability}

The model becomes richer if we introduce a threshold value $\delta$, corresponding to the rank above which it is more convenient for an agent to remain unmarried (or, similarly, not to buy a given product). In this situation one could wonder if it would be a better strategy to go down quicker on one's preference list, thus lowering one's probability of remaining single, or slower, in order to double one's chances of success with a better partner. Let us formalize this idea as follows: in a population of normal people (who propose to one partner at each 

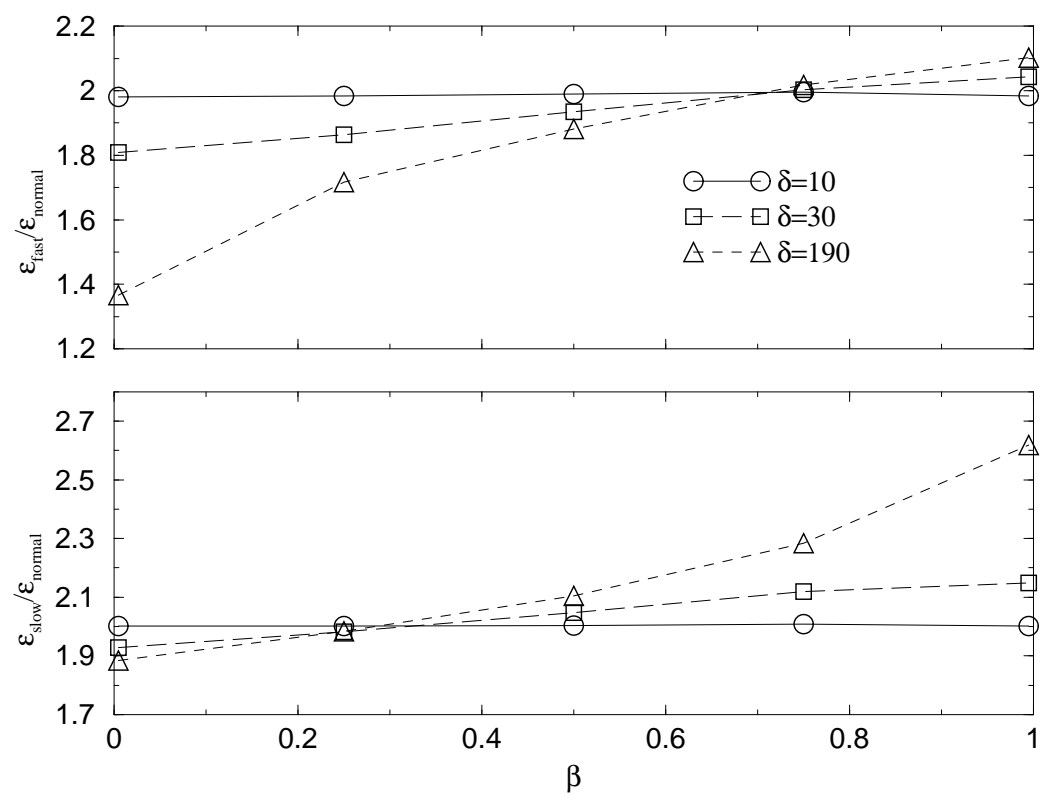

Fig. 3. Ratio between "fast" ("slow") people's energies and "normal" ones, as a function of the percentage of "normal" agents $\beta$. Symbols are simulation data for a 200 agents system with different thresholds $\delta$. Lines are inserted to guide the eyes.

time step) we can insert a percentage $(1-\beta)$ of "fast" (who propose to the next two agents of their preference list) or "slow" ones (who only make one proposal every two time steps). The game can thence be carried out as usual. Some agents will remain single at an energy cost $\delta+1$.

In fig. 3 we report simulation data from this model. There we analyze the ratios between the average energies of slow (fast) and normal people $\epsilon_{\text {slow(fast) }} / \epsilon_{\text {normal }}$. Both ratios remain bigger than one for any value of the percentage of normal people $\beta$. This proves that, given agents do not seek the optimal but the satisficing, it is always convenient to make only one proposal at each time step. In other words, we could make different possible strategies compete by Darwinian selection, giving each agent a number of offsprings proportional to her satisfaction at every generation. No matter the percentage of people holding a given strategy in the first generation, the FC one would always spread and succeed. In a wide sense the First Choice model meets, therefore, the requirements for evolutionary stability [20].

\section{Partial information}

Complete information is rare to be encountered in economic and social relations. Imperfect markets, where consumers do not even know the existence of some products, are more frequent than ideal ones. The FC model can be generalized to the case of limited information, that is people only know a portion 
$\alpha$ of the world. Accordingly, every preference list will contain $N(1-\alpha)$ holes. If people behaved according to the Gale-Shapley algorithm [1] the resulting satisfactions would be those reported in reference [9]. In the FC case agents propose, at time step $n$, to the $n^{\text {th }}$ ranked known counterpart player of their lists. Man $m$ could accept woman w's proposal only if $h(m, w)$ were smaller or equal to the rank of the $n^{\text {th }}$ known woman of his list. For small values of $\alpha$ some players find no partner and they remain single at an energy cost $\Delta_{c} \geq N$.

It is evident that information matters: the more people one knows, the more choice one has. It is then easy to state that $\epsilon$ must be a monotonically decreasing function of $\alpha$. In order to quantify this dependence, we notice that the average probability of accepting a proposal is of order $\epsilon / N$. If a player sent out $n$ proposals before finding her partner, the total probability $\epsilon \frac{n}{N}$ of getting married must equal unity (in the absence of singles). Starting from this observation we can follow the argument used in [2], which gives, in the symmetric case,

$$
\epsilon=\sqrt{\frac{N}{\alpha(2-\alpha)}} .
$$

A similar, yet more precise esteem, can be found as follows.

\subsection{Analytical estimation}

The probability $p_{n}$ that an agent gets married exactly at time step $n$ can be written as

$$
p_{n} \simeq 2 \frac{n}{\alpha N} q_{n-1}^{2}
$$

where $q_{n}$ is the probability of not being married and $n / \alpha N$ the average probability of accepting a proposal, at step $n$. In eq. (10), and in the following, we are neglecting: (i) The probability of having two agents proposing to each other at the same time. (ii)The probability of having two agents proposing to the same partner at the same time. The latter becomes important for $n_{\max }=N \alpha \simeq 1$. The number of singles diminishes with increasing $n$ according to the equation

$$
q_{n}=q_{n-1}-p_{n} \simeq q_{n-1}\left(1-2 \frac{n}{\alpha N} q_{n-1}\right),
$$

which can be solved for $\alpha \ll 1$, giving

$$
q_{n}=\left(1+\frac{n^{2}}{\alpha N}\right)^{-1}
$$




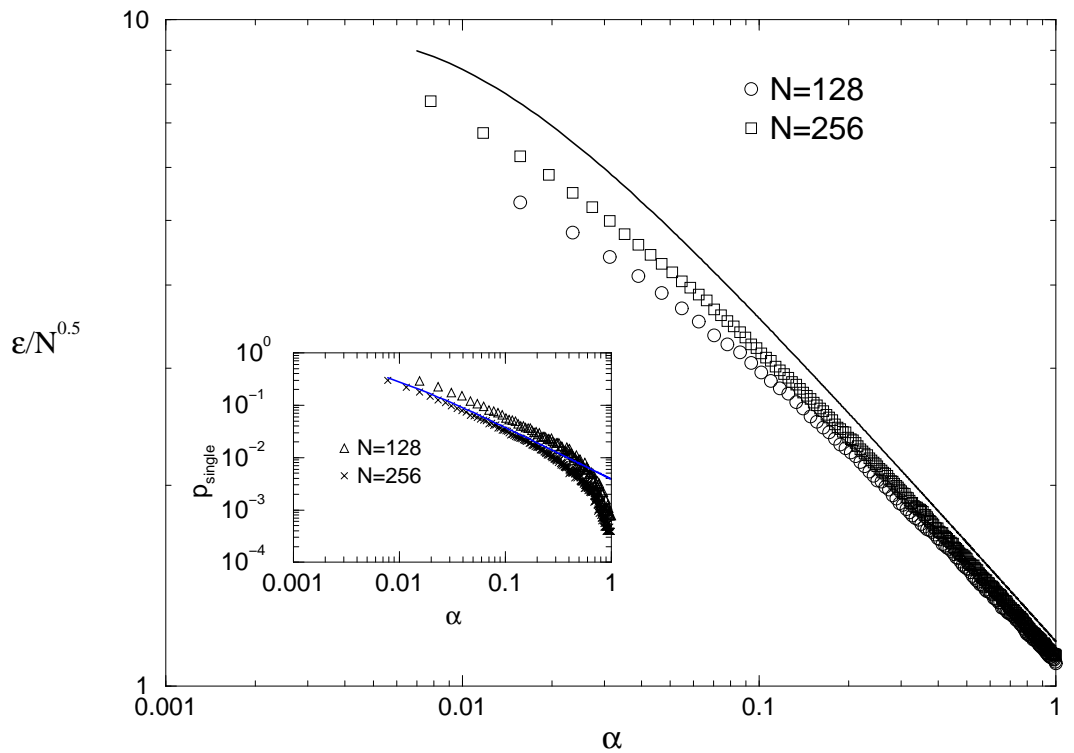

Fig. 4. Simulations of the FC method at increasing $\alpha$ values, with $\Delta_{c}=N+1$. Circles $(N=128)$ and squares $(N=256)$ are the average energies of agents as a function of $\alpha$, in $\log -\log$ scale. The solid line plots equation (13) for $N=256$. Inset: triangles up $(N=128)$ and asterisks $(N=256)$ are the percentage of singles, displayed in $\log -\log$ scale. The solid line plots $q_{\mathrm{N} \alpha}$ of equation (12) for $N=256$.

This means there are going to be singles even at the end of the FC process, i.e. for $n=\alpha N$. We compared such $q_{\alpha \mathrm{N}}$ with numerical simulations in the inset of figure 4 , finding a good agreement. When $\alpha=1$ there are no singles, and the exponential transition between the two regimes takes place around $\alpha \simeq \log N / N$, which corresponds to the analogous cut-off found in marriages with threshold [13].

Among the players who get married at time step $n$, half of them would be proposers and their energy would scale as $n / \alpha$; the other half would receive a proposal uniformly distributed between 1 and $n / \alpha$. Therefore the average energy of these agents reads $\epsilon_{n}=\frac{3 n}{4 \alpha}$. Inserting equation (12) into (10), we can calculate the average energy per agent as follows:

$$
\epsilon=\sum_{k=1}^{N \alpha} p_{k} \epsilon_{k} \simeq \frac{3 \pi}{8} \frac{N \sqrt{N \alpha}}{1+N \alpha}
$$

The above equation is plotted against $\alpha$ in figure 4 . Comparison with simulation data shows a better agreement for large $\alpha$ values than for smaller ones. This is probably due to the above mentioned fact that the neglected contribution of conflicting proposals becomes important when $n_{\max }=N \alpha$ is small. 


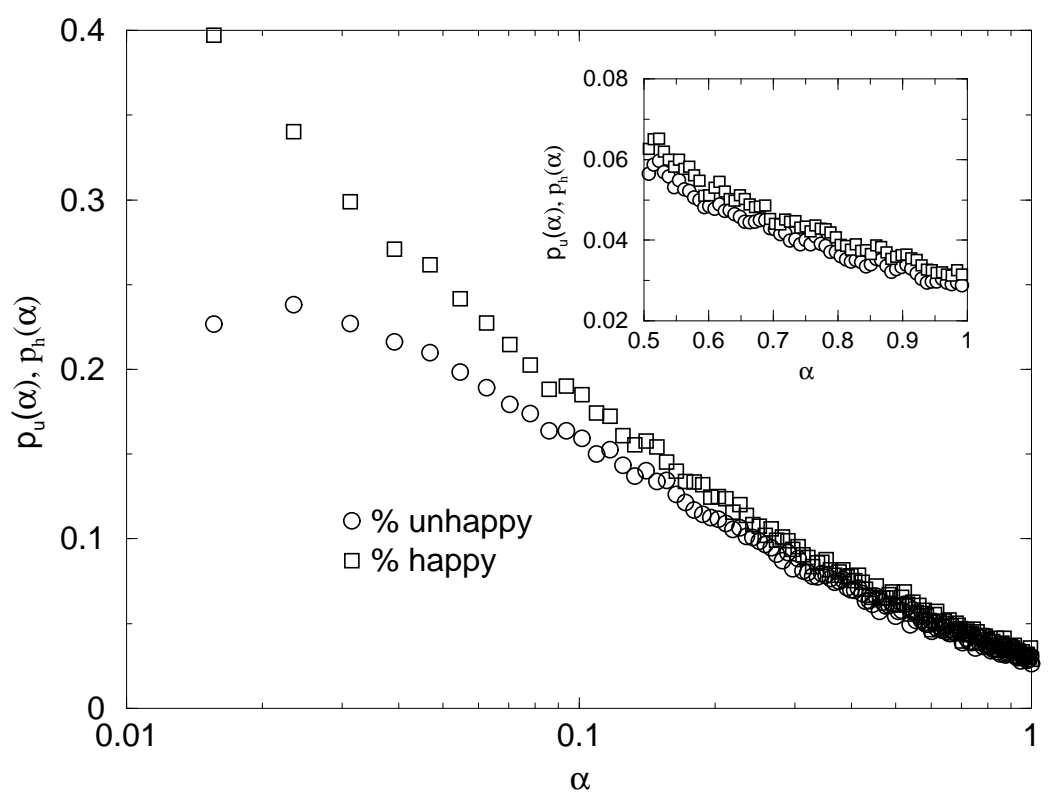

Fig. 5. Simulations of the FC method at increasing $\alpha$ values, with $N=128$. Circles are the percentage of unhappy people $p_{\mathrm{u}}$, squares that of happy ones $p_{\mathrm{h}}$, at constant increments $\Delta \alpha=1 / N$, plotted against $\alpha$ in semi-log scale. The inset highlights, in linear scale, the last half decade of the graph.

\subsection{Unhappy minority}

As $\alpha$ increases, the average energy per person drops very quickly. Now we ask ourselves how benefits are distributed in the population. In particular we shall focus our attention on the agents who do not benefit at all from an information increase. This kind of individual based analysis is crucial in economic systems, where the average data is not the end of the story.

We can imagine a situation where agents are endowed with a knowledge $\alpha_{1}$, find a FC matching $M_{1}$ with average energy $\epsilon\left(\alpha_{1}\right)$, and then acquire an additional amount of knowledge $\Delta \alpha$. This way players make new acquaintances and may find a better partner than they used to. The resulting new matching $M_{2}$ would correspond to a different point in the plot of fig. 4, i.e. $\epsilon\left(\alpha_{2}\right)$, where $\alpha_{2}=\alpha_{1}+\Delta \alpha$. The average energy decrease $\epsilon\left(\alpha_{2}\right)<\epsilon\left(\alpha_{1}\right)$ is due to the percentage $p_{h}(\alpha)$ of the population that improves its condition. On the other hand some players $\left(N p_{u}(\alpha)\right)$ suffer the couples reshuffling from $M_{1}$ to $M_{2}$, ending up with a higher energy.

We studied the percentage $p_{h}(\alpha)$ of "happy" people and that of unhappy ones $p_{u}(\alpha)$, at constant $\alpha$ increments $\Delta \alpha$. In figure 5 we reported the results for $\Delta \alpha=1 / N$. Notice that, in this case, the derivative $D_{u}(\alpha)=\lim _{\Delta \alpha \rightarrow 0} \frac{1}{N} \frac{p_{u}(\alpha)}{\Delta \alpha}$ if well estimated by $p_{u}(\alpha)$ itself, when $N$ goes to infinity. After a very short transient phase where the number of singles increases for the joint effect of 

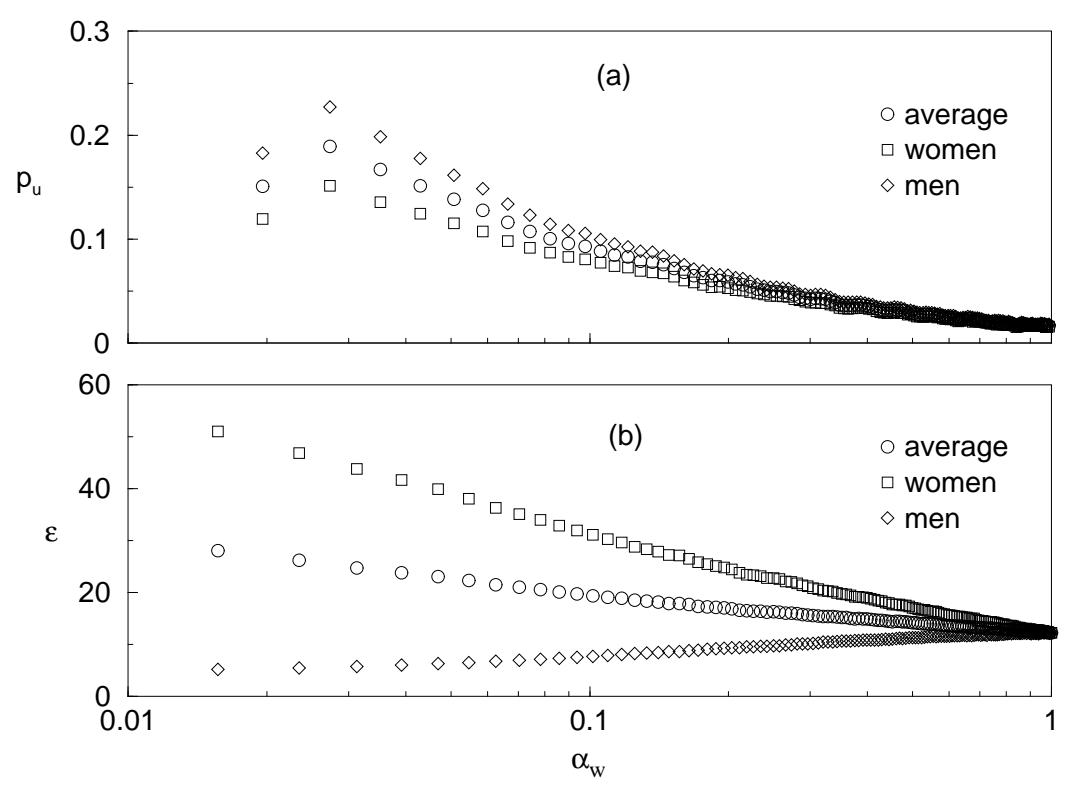

Fig. 6. Simulations of the FC method at fixed $\alpha_{\mathrm{m}}=1$ and increasing $\alpha_{\mathrm{w}}$ values, with $N=128$. The lower graph displays the average energy of agents (men, women and both) as a function of $\alpha_{\mathrm{w}}$, in semi-log scale. In the upper graph the corresponding percentage of unhappy people (men, women and both), at constant increments $\Delta \alpha_{\mathrm{w}}=1 / 128$, is plotted against the same log-scaled $\alpha_{\mathrm{w}}$ values.

competition and scarcity of alternatives, $p_{u}$ decreases logarithmically, and so does $p_{h}$. Notice that, even though the two percentages come closer as $\alpha$ is increased, $D_{h}(\alpha)$ stays always above $D_{u}(\alpha)$, as shown in the inset of figure 5 .

\subsection{Asymmetric information}

We have shown so far that information enhances the general satisfaction and damages slightly only a small minority of the population. Let us now give complete information to one side, say men $\left(\alpha_{\mathrm{m}}=1\right)$, and vary the other group's one $\left(\alpha_{\mathrm{w}} \in(0,1]\right)$. A similar situation occurs in economics when, for instance, firms have a much better knowledge of the market, that is their core business, than consumers do. Potential buyers, on the other hand, may increase considerably their amount of information by gathering sellers' reputation data, which becomes easily feasible in the Internet age [6].

In figure 6.a we plotted the probability of being unhappy $p_{u}\left(\alpha_{\mathrm{w}}\right)$, with the same definition as before, for men, women and both. The behavior is very similar to the symmetric case. As expected, there are more men than women suffering from the unilateral injection of information in the game. Here men are justly paying the price of equal rights.

In figure 6.b the corresponding energies of both sexes are plotted against $\alpha_{\mathrm{w}}$ 
in semi-log scale. It appears clearly that women's energy (squares) decreases while men's energy (diamonds) increases, as $\alpha_{\mathrm{w}}$ grows. Then men's satisfaction deteriorates, even if their information does not change during the process. Nevertheless the general average energy, represented by open circles in figure 6 , diminishes. In other words men's loss is more than compensated by women's improvement: even a unilateral information increase is beneficial to the society as a whole. This confirms the pie augmentation hypothesis made in [2].

\section{Matchmaker}

When information is only partial players score very poorly (see fig. 4). In some circumstances it could be convenient for them to let someone else find them a partner, rather than doing it by themselves. This becomes possible -and even likely - in a world where the connectivity distribution of human social contacts is very broad [21]. More than that, in the Internet age very few highly connected nodes [22] could gather a huge amount of information.

Let us imagine that people in a community agree to give their preferences lists to a matchmaker (MM)[2]. He possess now complete information and is capable of finding the Ground State of the system. Accordingly, he proposes each player a partner: if everybody accepted the MM's proposal, then the community would minimize the total energy. Nevertheless we assume players are selfish and careless of the general welfare. Thus they keep looking for a better partner among those players whom they know, employing their maximum searching effort. If a man $m$ and a woman $w$ prefer to marry one another rather than accepting the MM's offer, then they refuse it, step out of the community and get married. Such a decision forces in the meantime two more players ( $m$ 's and $w$ 's partners in the Ground State) to look for a new partner. For each value of $\alpha$ there would be a percentage $p_{i}(\alpha)$ of such "independent" people.

A rough esteem of $p_{i}$ can be obtained by calculating the probability that an individual FC energy is lower than that of the Ground State. To do that, we shall assume that the distribution of individual energies in the FC state $p^{F C}(x, \alpha)$ equals that of the OSS (eq. (7)) for $\alpha=1$, and that $p^{F C}(x, 1) d x=$ $p^{F C}(x / \sqrt{\alpha}, \alpha) d x / \sqrt{\alpha}$. These approximations are only reasonable when $\alpha \gg$ $\alpha_{c}$. If we take equation (2) for the individual energy distribution in the Ground State, we obtain:

$$
p_{i}(\alpha) \propto\left[\sqrt{\alpha} \int_{0}^{\sqrt{N}} d x e^{-x} \int_{0}^{x} d y e^{-0.3 y(2+y)}\right]^{2} \simeq 0.2 \alpha
$$



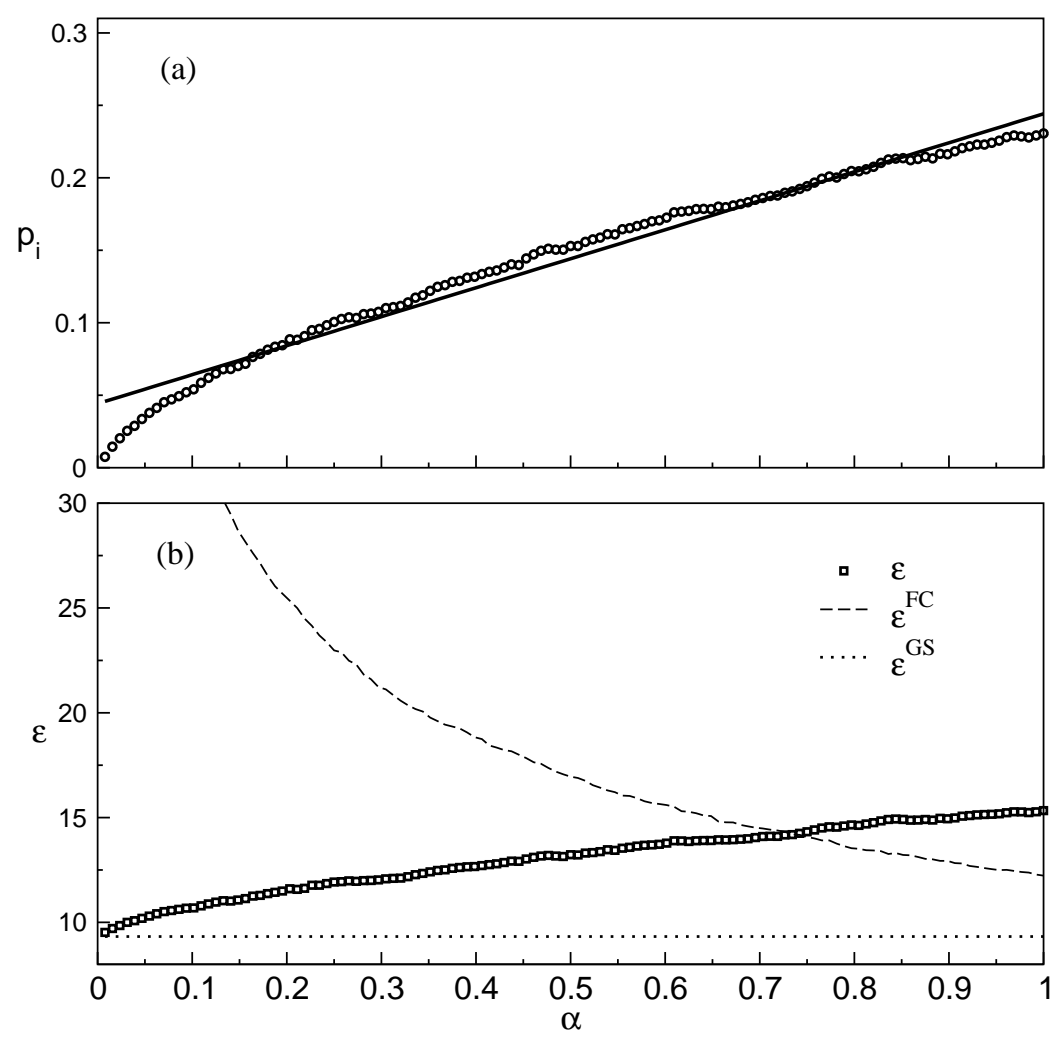

Fig. 7. Simulation data of a Marriage game with $N=128$ players for each gender. Averages are taken over $10^{4}$ realizations for each value of $\alpha$, whose $\mathrm{x}$-axis linear scale is the same for all graphs. (a)Circles represent the percentage $p_{\mathrm{i}}$ of independent people. The solid line plots equation (14). (b)Squares represent the overall average energy per person $\epsilon$. The dotted line is the value of the Ground State energy, corresponding to $p_{\mathrm{i}}=0$, the dashed line that of the FC energy.

for $N$ approaching to infinity. In figure 7.a equation (14) is compared with simulations, and the agreement seems good.

In figure 7.b the overall average energy per person $\epsilon$ is plotted against $\alpha$. Its lower and upper bounds should respectively be the Ground State energy (displayed as dotted line) and the FC one (displayed as dashed line). It is clearly not the case for the latter, because half of the independent couples are rematched randomly, having been forced to divorce by their mates. The crossing point (around $\alpha=0.72$ ) corresponds, in the graph 7.a, to a critical percentage of independent people. Above that value (about $2 \%$ ) the MM can no more be useful, unless he employed sophisticated mechanisms. For instance he could find the matching of minimal energy among the "loyal" people; but this could become an endless process, since it would generate a new cascade of desertions, and so forth.

For sure, at least below that critical value, $\epsilon$ is a monotonically increasing function of $\alpha$. This means that people with partial information (small $\alpha$ ) can score better than those with infinite searching power $(\alpha=1)$, given that they 
renounce to their personal privacy. This effect would be amplified by the fact that some of them would find the MM's offer satisficing, and not search any further for a different partner. In other words what we are showing in fig. 7.b is the worst case of anti-social behavior at any given average information level. In real life very few people would employ all their searching effort looking for a substitute good (partners might be an exception) if they were satisfied enough with the one they already have [4]. This way most of the people will remain in the MM's community, thus saving searching costs and being better off on average. Such savings can be thought of as the MM's revenue. The above argument becomes more effective if we imagine that the game be repeated numerous times, in which case reputation matters [6]. Then people would care more about their average utility and might be pleased by a long lasting confidence relationship with the MM.

Of course we should consider the possibility of a corrupted MM, who would favor some players at the expense of the collectivity. However, the matchmaker institution would survive only if the average energy of those who accept his proposals is lower than that of those who found a partner on their own. Moreover, when the information that players release to the MM is not used properly, it would start to be convenient for them to protect their privacy. In such a situation it's no more profitable for the MM to be corrupted and an equilibrium is likely to arise. By construction, at the equilibrium point the average satisfaction is bigger than it would be without the MM, and more evenly distributed (the variance of the Ground State distribution, plotted in the inset of fig. 1, is the smallest). Furthermore, we should consider competition among different MMs, in which case a cheating one would be exposed to the danger of loosing clients and lower his earnings.

\section{Market implications}

A real market could be thought of as a polygamic marriage game with unequal number of players. In order to point out the role of information, we can try to carry on this metaphor letting rational agents with partial information play the game.

Let us consider a market with $N_{c}$ consumers and $n$ enterprises, such that $n \ll N_{c}$, where every enterprise produces the same type of good. Enterprises have a completely degenerate preference list, i.e. they make no discrimination among different consumers. Each enterprise $i$ is endowed with a budget equal to 1 , a fraction $\left(1-q_{i}\right)$ of which will be invested in the marketing process, a fraction $q_{i}$ in the quality of the product. Here "quality" includes the research expenditures and the costs of production. Consumers act as in the FC model. They get to know the different brands through commercials, plus an effort 
$e_{k} \in(0,1)$, which represents the amount of personal search. $e_{k}=1$ corresponds to disposing of and processing infinite information, $e_{k}=0$ to no information effort. The probability $\alpha_{k}(i)$ that consumer $k$ knows the product $i$ should be a convex function of $\left(1-q_{i}\right)$; for the sake of simplicity, let us assume it linear:

$$
\alpha_{k}(i)=\min \left\{1,1-q_{i}+e_{k}\right\}
$$

On the other hand, for consumer $k$ the product $i$ has an expected utility

$$
u_{k}(i)=q_{i}+\eta_{k}\left(1-q_{i}\right)
$$

where $\eta_{k}$ is a random variable, uniformly distributed between zero and one. This way consumers have a perception of the quality of the goods $\left(q_{i}\right)$, but commercials $\left(1-q_{i}\right)$ are aimed to confuse them, so that the products look nicer than they really are. In fact information is not only limited in real life, but it is also imperfect $[2,6]$. The factor $\eta_{k}$ is intended to account for commercials more or less effective, for different tastes and needs of consumers. Higher expected utility corresponds to better ranking in one's preference list. This approach resembles the "beauty correlated" marriage problem, studied in a recent paper [19]. All products have the same price, but different qualities.

At each instance of the game consumers buy one unit of good. In order to maximize their expected utility, they choose the best ranked among those they know of. As a first, simple example, let us set up a game with the variables $q_{i}$ randomly distributed between zero and one. Each consumer knows an average of $r(e)=n(1+2 e) / 2$ different products if the average effort $e$ is smaller than $0.5, n$ products otherwise. Consumers' average utility is a monotonically increasing function of $e$ and $n$. Now we introduce a new enterprise and ask ourselves the $q$ value $\hat{q}$ that maximizes its selling. With some approximation we can show that the probability of being the best ranked, among the $r(e)$ that each consumer knows, has the form $p_{\text {best }}(q, r) \simeq \frac{\left(1-q^{r-1}\right)}{(1-q) r}$. Multiplying $p_{\text {best }}$ and $\alpha_{k}(i)$ (eq. 15) we find the average selling of the new enterprise:

$$
s(q, e)=N_{c}(1-q+e) \frac{\left(1-q^{n \frac{1+2 e}{2}-1}\right)}{(1-q) n \frac{1+2 e}{2}}
$$

For $e<0.5$ we can assume $r \simeq n / 2$. With this position we find that the maximum of the above equation lies on the curve

$$
e(\hat{q})=\frac{(n-2)(1-\hat{q})^{2} \hat{q}^{n / 2}}{2 \hat{q}^{2}+\hat{q}^{n / 2}[\hat{q}(n-\hat{q})+2-n]},
$$

that is a monotonically increasing function of $\hat{q}$. This means that, as expected, the optimal fraction of quality investment $\hat{q}$ increases as the consumers' effort 


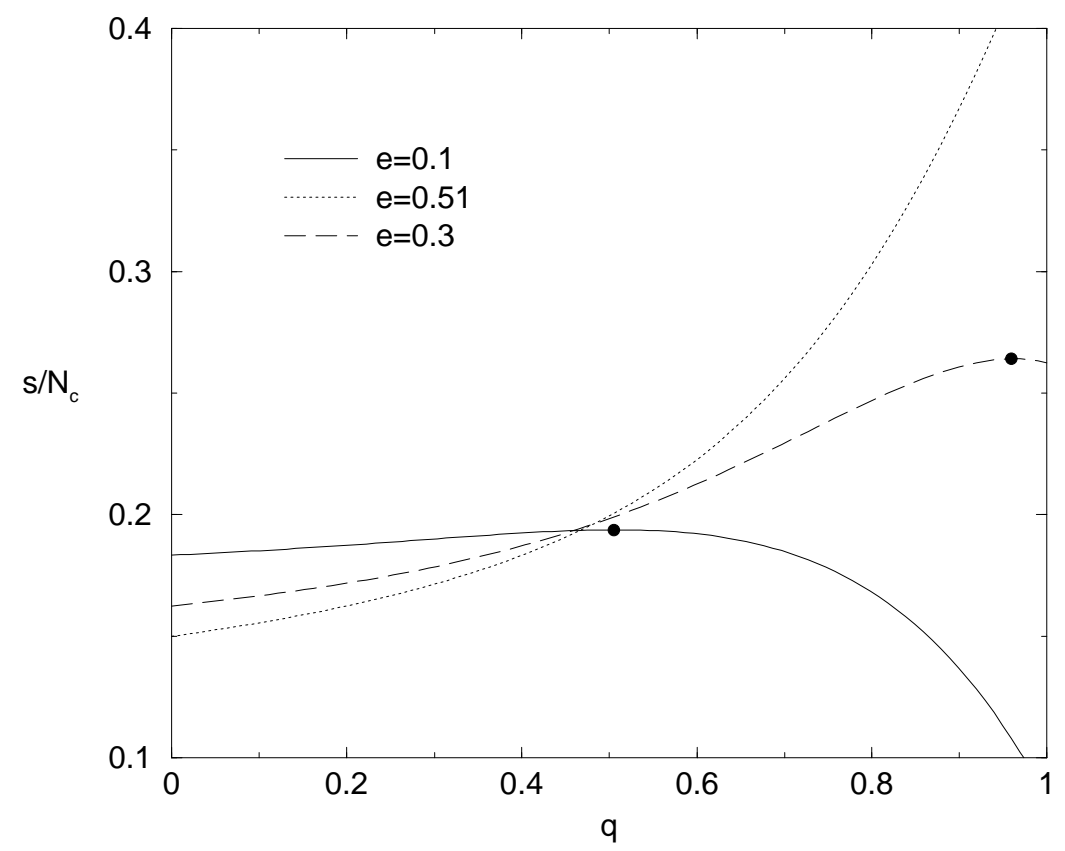

Fig. 8. Probability of selling an item as a function of the quality investment $q$. The plots represent eq. (17), with $n=10$, for three different values of the average consumers' effort $e$. Filled points are the maxima $\hat{q}$ of the curves for $e=0.1$ and $e=0.3$. For $e=0.51 s$ is an increasing function of $q$, therefore $\hat{q}=1$.

grows (see fig. 8). In other words, the bigger portion of the market consumers know, the more enterprises are likely to invest in the quality of the product. Moreover, the amount of effort needed to make it convenient to the new enterprise to invest all its capital in the quality, $e(\hat{q}=1)=\frac{4}{n-4}$, diminishes sensibly with increasing $n$. Similar arguments hold for $e>0.5$.

At a given value of the consumers' effort $e$, some enterprises would adjust their $q$ investment to exploit the edge left by the other ones. But how do consumers decide their searching effort level? Increased computing power may allow for increasing information capability with no additional cost. Still, some information is costly [23]. One way to take care of it is subtracting the search costs to the expected utility. In this more realistic case consumers may decide how much they want to invest in searching a good, given the average quality of the products. In the scenario we pictured above, they would probably search until they are satisfied, without necessarily maximizing their utility function. In fact, what is a core business for an enterprise is a marginal one for most consumers. For this reason, even if some enterprises had a perfect knowledge of the market and could find their $\hat{q}$ at any time, it would always be convenient for them to chose a lower level of quality investment. 


\section{Acknowledgments}

We would like to thank A. Capocci and Joseph Wakeling for useful comments. This work was supported by the Swiss National Fund, Grant No. 20-61470.00.

\section{References}

[1] D. Gale and L.S. Shapley L.S., Am. Math. Monthly, 69 (1962) 9.

[2] Y.C. Zhang, Physica A, 299 (2001) 104.

[3] J. Nash, Proc. Nat. Acad. Sci. 36, 48 (1950).

[4] H. Simon "Theories of Bounded Rationaliuty." In, Models of Bounded Rationality. Behavioral Economics and Business Organization. Pp. 408-423. Vol. 2. Cambridge: MIT Press, 1982.

[5] G.A. Akerlof, Quarterly Journal of Economics, 84 (1970) 488.

[6] P. Laureti, F. Slanina, Y.-K. Yu, Y.-C. Zhang, Physica A, 316 (2002) 413.

[7] T.C. Schelling, Micro Motives and Macro Behavior. New York, W.W. Norton \& Company, 1978

[8] B. Pittel, Ann.Appl.Prob., 2 (1992) 358.

[9] G. Caldarelli, A. Capocci, P. Laureti, Physica A, 299 (2001) 268.

[10] D.E. Knuth, Mariages Stables. Les Presses de l'Université de Montréal, Montréal, 1976.

[11] D. Gusfield, R.W. Irving, The Stable Marriage Problem: Structure and Algorithms. The MIT Press Cambridge, Massachusetts, 1989.

[12] R. W. Irving, P. Leather, SIAM J. Comput., 15 (1986) 655.

[13] M. Dzierzawa and M.J. Oméro, Physica A, 287 (2000) 321.

[14] R. W. Irving, P. Leather, and D. Gusfield, Journal of the ACM, 34 (1987) 532.

[15] D. Sleator, R.E. Tarjan, J. Comput. Syst. Sci., 26 (1983) 362.

[16] M.-J. Omero, M. Dzierzawa, M. Marsili and Y.-C. Zhang, J. Physique I France, 7 (1997) 1723.

[17] C. H. Papadimitriou and K. Steiglitz. Combinatorial Optimization: Algorithms and Complexity. Prentice-Hall, 1982.

[18] H.W. Kuhn, Nav. Res. Logist. Quart., 2 (1955) 83.

[19] G. Caldarelli, A. Capocci, Physica A, 300 (2001) 325. 
[20] G. M. Smith, Evolution and the theory of games, Paperback (1982)

[21] F. Liljeros, C.R. Edling, L.A.N. Amaral, H.E. Stanley, Y. Aberg, Nature 411 (2001) 907.

[22] C. Faloutsos, P. Faloutsos, M. Faloutsos, In Preceedings of the ACM SIGCOMM September 1999.

[23] G. Stigler, Journal of Political Economy, 69, (1961) 213. 